SCIREA Journal of Clinical Medicine

ISSN: 2706-8870

http://www.scirea.org/journal/CM

April 26, 2021

SCIREA

Volume 6, Issue 3, June 2021

\title{
CASCADE STOMACH - COMMENTARY A PROPOS A CASE
}

\section{Daniel Benharroch ${ }^{1}$, Roberto Umansky ${ }^{2}$}

${ }^{1}$ Department of Pathology, Soroka University Medical Centre, and Faculty of Health Sciences, Ben Gurion University of the Negev, Beer Sheva, Israel.

${ }^{2}$ Psychiatrist, Independent Physician, Retired. Beer-Sheva, Israel.

Correspondence: Prof. Daniel Benharroch, Independent Physician, Soroka University Medical Centre, 151 Rager Blvd, P.O.Box 151, Beer-Sheva, 84101, Israel. Cell 972507579140. E-mail: danielbenharroch1@gmail.com

\begin{abstract}
The recognition of a cascade stomach, based on imaging, is not usually disputed by the clinician. Except for initiating therapy for reflux symptoms or for dyspepsia, a specific treatment is seldom required. Thus, a cascade stomach rarely attracts the interest of the gastroenterologist. Occasionally, more disparate features are described in a patient displaying a radiological upper gastroenterological series of this condition. It remains to prove that such symptoms represent coincidental, but non-specific features of the ailment. A patient diagnosed with a cascade stomach, who exhibited various upper abdominal complaints, the majority of which are not associated, as a rule, with this condition, is hereby reported.
\end{abstract}

Keywords: cascade stomach; dyspepsia; reflux symptoms; distal esophageal spasm 


\section{INTRODUCTION}

A cascade stomach is mostly diagnosed using an upper gastrointestinal series. A contrast material (barium) is swallowed by the fasting patient and is traced by fluoroscopy, to disclose the esophagus, stomach and duodenum. This "variant" stomach pathophysiology bears several forms [1]. However, the demonstration of an enhanced angulation with an air-fluid level encompassing the stomach, proximally to the angulus, is required [2]. Moreover, the fundus, often almost empty, or showing an additional air-fluid level, will exhibit parallel linear tracks of the contrast material, that highlights the unmarked gastric folds - the "cup and spill" [3].

The symptoms, generally considered to accompany this condition, are scarce and have included mainly dyspepsia and gastroesophageal reflux features [3]. Dysphagia has also been rarely described [4]. Additional studies have noted further characteristics, mentioned as "obscure" by one author [5]. There should be a way to confirm the association of these "atypical" symptoms with the disease entity. If the indefinite symptoms are shown to belong formally with the cascade stomach, one might not assess this relationship as incidental [5]. Modes of therapy, both medical and surgical have been previously suggested. However, is treatment justified in these cases [6]? A patient with this disease entity is presently reported.

\section{CASE PRESENTATION}

Following the recurrence of ischio-rectal abscesses, this 24-year-old soldier was submitted to an upper gastrointestinal series. The radiological exam did not display the existence of an anorectal fistula, as evidence against the alleged Crohn's disease. Instead, it highlighted the presence of a cascade stomach (Fig. 1 a. \& b.).

The subject had complained of epigastric pain, after meals and of heartburns, from the age of 16, but for a long period of time, was neither diagnosed, nor treated. Progressively, the symptoms evolved, to crystalize into a gastroesophageal reflux. At the age of 46, the patient was submitted to a urease test, which disclosed a $\mathrm{H}$. pylori infestation. Otherwise, the gastroscopy was unremarkable.

Soon after treatment with three agents for the H. pylori infection, the patient was free from heartburns. However, following persistent and annoying aerophagia and meteorism, he was diagnosed with lactose intolerance. Several gastroscopies had shown normal features, except, 
intermittently, for evidence of a mild gastroesophageal reflux. Two of the exams revealed prepyloric, low grade intestinal metaplasia.

The individual had led a 'relatively' comfortable life, till the age of 71. By now, he had started complaining of dysphagia, with difficulty in swallowing mainly water. He also described short runs of hiccup, especially at raising from bed, and a few minutes after meals, in addition to belching.

Several investigations were ordered, in addition, most of which were GI-related. A few were directed toward musculoskeletal symptoms. Except for the suspicion of distal esophageal spasm (DES), all were about normal.

A cardiologist discussed a mild to moderate aortic regurgitation, as an isolated finding, at the completion of his investigations. An ORL exam was within normal limits. Nevertheless, the patient was referred for esophageal manometry. The manipulation raised the suspicion of a distal esophageal spasm (DES) (Fig. 3). The patient was prescribed esomeprazole, $20 \mathrm{mg} / \mathrm{d}$ for a month, as his symptoms did not bear the severity usually attributed to achalasia. It was felt that the various ailments, might not all derive directly from the cascade stomach (Fig. 2); especially, not after a long symptomless period had evolved.

Due to a discrepancy, by which the patient described a swallowing difficulty localized to the proximal part of the esophagus, the gastroenterologist consulted again the patient's files. This physician noted that, at 16 years of age, the subject had been diagnosed with pulmonary tuberculosis. Among the three medications he was prescribed for this infection, for a 9 month duration, para-amino-salicylic acid (PAS) was provided in the form of 12 tablets, to be swallowed every morning.

The gastroenterologist related that, recently, the patient had been prescribed aripiprazole $5 \mathrm{mg}$, once daily for the maintenance treatment of a long standing, otherwise well-managed, schizoaffective disorder. At the lecture of the package insert, he noticed further that the medication contained $26 \mathrm{mg}$ lactose per dose; that it might cause difficulties in swallowing, in addition to abdominal and gastric discomfort, weight gain, increased salivation, as well as rapid breathing with a difficult respiration. The leaflet mentioned among others, sequels for special attention, difficulty in swallowing with occasional inhalation of fluids and food, and potentially, subsequent pneumonia. Most of the above were comprised among the symptoms the patient had reported, at one time or other, even though the dose of aripiprazole was the lowest possibly accounted for. 
The patient's psychiatrist has searched persistently for an appropriate, new generation antipsychotic drug, but, using trial and error, he met time and again, with unacceptable adverse effects. A search for a more admissible candidate drug is ongoing.

\section{DISCUSSION}

A 71-year-old man is presented with a complex gastrointestinal array of symptoms, fortyseven years after the incidental diagnosis of cascade stomach, during which time he had suffered of heartburn and of intermittent epigastric pain only. Endoscopy had not been contributory [7]

Among his past maladies, tuberculosis at age 16 and a schizoaffective, bipolar disorder, diagnosed at age 28, and appropriately managed, were accounted for.

The patient's saga rises two main queries: primarily, was the cascade stomach, idiopathic, or perhaps, a cascade-like defect consequent to the massive and extended PAS therapy at 16, age marking the outset of his GIT complaints? No indication of a causal effect between PAS and a gastric pathology of any type has been retrieved, although this medication, especially if administered in the form of tablets, had not been without consequences [8]. This drug is now used as a liquid form, both per os and by injection, including for drug resistant tuberculosis.

Moreover, a cascade stomach is usually described in conjunction with reflux symptoms or with dyspepsia and rarely with dysphagia. Anyway, what could be the cause of the clustering of new GIT ailments, many years after the diagnosis of the cascade defect?

An expensive array of studies, including CT scans, a MRI, and an esophageal manometry, were interpreted as normal or near normal.

The culprit might well be identified in regard with the second query!

The second issue concerns the severe sequels due to aripiprazole, which afflicted the present patient. These complications might result in distress, in an unknown proportion of psychotic patients [9]. Do the (atypical) antipsychotic medications (AADs) alleviate, in any way the suffering of these individuals, or do they make things worse for them. The poor compliance shown in more than a fifth of the patients in some series, as well as their dropping-out of follow-up, may favor this opinion [10-12]. However, psychotic patients often fail to report the serious sequels of the AADs. Their physicians, having performed their duty, by the book, are relieved, at least temporarily, from their responsibility. 
Conclusion: This report has highlighted two sets of evidence, the first being that cascade stomach is still a poorly understood condition and that all the symptoms that may accompany it, are not necessarily an integral part of the illness. Moreover, we have confirmed a truism: that administration of the AADs to patients with schizoaffective disorders, may ameliorate significantly their condition. However, the price, in terms of complications, might be so high, that their compliance drops by more than $20 \%$, often to the point of their abandoning the treatment and at times, the follow-up as well.

\section{ACKNOWLEDGEMENTS}

We thank Kibbutz Sde Boker for their support.

The patient has given his written approval for the report of his medical history.

The authors have declared 'No conflict of interests exists'.

No funding was necessary for this report.

\section{FIGURE LEGENDS}

Fig. 1. The patient's upper gastrointestinal fluoroscopy discloses an increased angulation with an air-fluid level at the gastric fundus (Fig. 1a). Note the emphasis on the folds (by negative contrast) of the gastric body, evoking a waterfall (Fig. 1b).

Fig. 1a

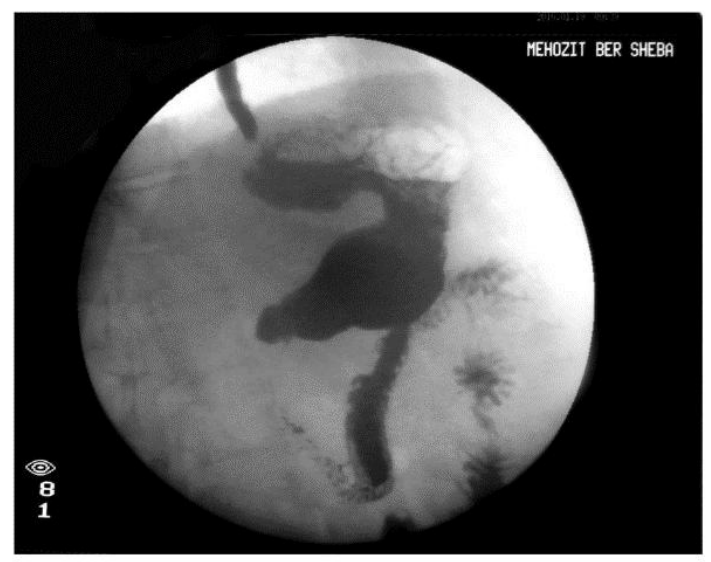

Fig. 1b

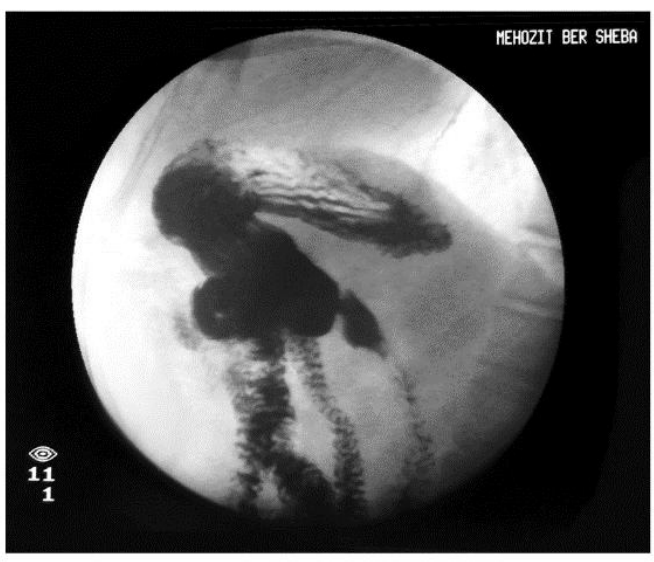




\section{REFERENCES}

[1] Burdan F, Rozyla-Kaliniwska I, Szumilo J, et al. Anatomical classification of the shape and topography of the stomach. Surg Radiol Anat. 2012;34:171-178.

[2] Keller RJ, Khilnani MT, Wolf BS. Cascade stomach, roentgen appearance and significance. AJR. 1975;123:746-754.

[3] Bradsbury C, Balaji N, The cascade stomach. Revisited in the $21^{\text {st }}$ century - what has changed? Poster, University Hospitals in the North Midlands, UK. 2017.

[4] Kusano M, Hosaka H, Moki H, et al. Cascade stomach is associated with upper GI symptoms, a population-based study. Neurogastroenterology Motility. 2012;24:451-455.

[5] Gulsen MT, Koruk I, Dogan M, et al. Diagnostic accuracy of cascade stomach by upper GI endoscopy in patients with obscure symptoms: A multi-center prospective trial. Clin Res Hepatol Gastroenterol. 2011;35:171-178.

[6] Chhabra MK, Mongia AK. Cascade stomach: a case report. Int Surg J. 2016;3:1005-1008.

[7] Kusano M, Hosaka H, Yasuoka H, et al. New endoscopic classification of cascade stomach, a risk factor for reflux esophagitis. J Gatroenterol. 2017;52:211-217.

[8] Zumstein P. Side effects of para-aminosalicylic acid (PAS). Praxis. 1956;45:48-56.

[9] Brown R, Taylor MJ, Geddes J. Aripiprazole alone or in combination with acute mania. Cochrane Database System Review. 2013;17(2): CD005000.

[10] Flanagan RJ. Side effects of clozapine and some other psychoactive drugs. Curr Drug Saf. 2008;3:1155-22.

[11] Trenton A, Currier G, Zwemer F. Fatalities associated with therapeutic use or overdose of atypical antipsychotics. CNS Drugs. 2003;17:307-324.

[12] Chaumartin N, Monville M, Lachaux B. Dysphagia or dysphagias during neuroleptic medication. Encephale. 2012;38:351-355. 